\title{
INFLUENCIA DE LA RESPONSABILIDAD SOCIAL EMPRESARIAL EN LA IMAGEN INSTITUCIONAL DE LAS ENTIDADES FINANCIERAS DE TACNA, AÑO 2017.
}

\author{
INFLUENCE OF CORPORATE SOCIAL RESPONSIBILITY IN THE INSTITUTIONAL IMAGE OF THE \\ FINANCIAL INSTITUTIONS OF TACNA, YEAR 2017.
}

\author{
Omar Julinho Hidalgo Aguilar ${ }^{1}$ \\ Presentado: $18 / 08 / 2018$ \\ Aceptado: $17 / 09 / 2018$ \\ Publicado online:27/12/2018
}

\section{RESUMEN}

El presente trabajo de investigación tiene como objetivo principal el determinar en qué medida la responsabilidad social empresarial influye en la imagen institucional que perciben los clientes de las entidades financieras de Tacna, año 2017. Estudio de tipo básico y correlacional, de diseño no experimental. La muestra fue de 381 clientes de las instituciones financieras: Banco de Crédito del Perú, BBVA Continental, Scotiabank e Interbank. Se encontró que la responsabilidad social empresarial influye en la imagen institucional que perciben los clientes $(p=0,010)$; el balance económico no influye en la imagen institucional que perciben los clientes $(p=0,209)$; el balance social influye en la imagen institucional que perciben los clientes ( $p$ $=0,048)$; y el balance ambiental no influye en la imagen institucional que perciben los clientes ( $p=0,749)$. El $57,9 \%$ de los clientes considera que es de nivel regular la responsabilidad social empresarial que perciben de las entidades financieras de Tacna, y el $61,2 \%$ de los clientes considera que es de nivel regular la imagen institucional que perciben de las entidades financieras.

Palabras clave: Responsabilidad social empresarial, imagen institucional.

\section{ABSTRACT}

The main objective of this research work is to determine the extent to which corporate social responsibility influences the institutional image perceived by customers of Tacna financial institutions, year 2017. Study Basic and correlational type, non-experimental design. The sample consisted of 381 clients from financial institutions: Banco de Crédito del Perú, BBVA Continental, Scotiabank and Interbank. It was found that corporate social 
responsibility influences the institutional image perceived by clients $(p=$ 0.010); the economic balance does not influence the institutional image perceived by clients ( $p=0.209$ ); the social balance influences the institutional image perceived by clients $(p=0.048)$; and the environmental balance does not influence the institutional image perceived by clients $(p=$ 0.749). $57.9 \%$ of the clients consider that the corporate social responsibility they receive from Tacna financial institutions is of a regular level, and $61.2 \%$ of the clients consider that the institutional image they receive from the entities is of a regular level. Financial

Keywords: Corporate social responsibility, institutional image.

\section{INTRODUCCIÓN}

El trabajo de investigación tuvo como objetivo principal el determinar en qué medida la responsabilidad social empresarial influye en la imagen institucional que perciben los clientes de las entidades financieras de Tacna, año 2017; puesto que uno de los principales objetivos de los bancos es impulsar que las condiciones de vida donde desarrollan sus transacciones financieras sean adecuadas, para que sus clientes puedan generar ingresos que les permitan atender los compromisos asumidos con las entidades financieras. A nivel internacional, existe una tendencia a que cada vez más las organizaciones prioricen el fortalecer sus funciones de responsabilidad social, puesto que son conscientes de que es importante la contribución activa y voluntaria en el mejoramiento de las actividades vitales de la sociedad, principalmente a través del buscar financiar proyectos que se caracterizan por su elevado contenido social o medio ambiental. En el Perú, el sector financiero es uno de los sectores más dinámicos que se preocupa por dar un servicio de calidad a sus clientes, pero en lo que respecta a impulsar actividades de responsabilidad social está recién buscando priorizarlas; sobre ello Lizarzaburu y Del Brío (2016) precisa que desde inicios del siglo XXI, el sector financiero peruano ha empezado a incluir en sus planes estratégicos, lo concerniente a estrategias de Responsabilidad Social Corporativa - RSC, puesto que han encontrado que dichas acciones están permitiendo que su reputación como entidad mejore. Las organizaciones a medida que buscan ser más competitivas en el mercado, consideran que el ser responsables socialmente es un aspecto vital para recibir el respaldo de la sociedad respecto a la calidad del producto $\mathrm{y} / \mathrm{o}$ servicio que proporcionan (Castillo, 2005). El principal desafío para una entidad financiera en el tema de la responsabilidad social, es el fortalecer la reputación de la organización aumentando la percepción de valor atribuida por sus grupos de interés (Marquina, 2015).

Pérez - Aranda (2016) concluye que es prioritario incorporar la teoría del desarrollo sostenible en la explicación de la responsabilidad social, puesto que los clientes de cadenas de hoteles logran asociar la dimensión ambiental como elemento clave en el desarrollar la responsabilidad social. Marquina (2015) encontró que la probabilidad de compra de los consumidores de zapatillas aumenta si la empresa fabricante cumple con determinadas características en su gestión: compromiso con el medio ambiente, buen trato a los trabajadores, apoyo a programas de lucha contra la pobreza, calidad de los productos y oferta de productos atractivos e innovadores; Cea (2010) propone un modelo normalizado de Informe de RSC/Sostenibilidad para las entidades bancarias donde se logra concluir que la acciones que priorizan las entidades bancarias sobre la responsabilidad social, no son motivadas solamente por causas altruistas orientados a mejorar los impactos sociales y 
ambientales, sino que también obedece a motivos de rentabilidad financiera. Alvarado y Schlesinger (2008) desarrollaron una investigación denominado "Dimensionalidad de la Responsabilidad Social Empresarial Percibida y sus Efectos sobre la Imagen y Reputación: Una Aproximación desde el Modelo de Carroll"; de tipo básico de nivel correlacional, de diseño no experimental; siendo la muestra de 358 consumidores jóvenes de telefonía celular de la ciudad de Valencia (España); y se concluye que las acciones y programas de responsabilidad social empresarial podrían mejorar la imagen y la reputación de las empresas ante los ojos de sus consumidores y ser una importante fuente de ventaja competitiva para las mismas, además los resultados indicaron que cada una de las dimensiones de responsabilidad social influyen significativa y directamente sobre la imagen de la marca, e indirectamente sobre la reputación. Mercado y García (2007) en México, encontró que las empresas se encuentran en el nivel inicial de filantropía, detectándose la voluntad de la alta dirección y de los accionistas como factores que podrían favorecer la adopción de la responsabilidad social. Marquina (2012) en Perú, encontró que existe una relación positiva entre la responsabilidad social empresarial y el comportamiento de compra, y que su efecto en conjunto es superior al de las competencias corporativas. Quihue (2011) en Tacna, Perú concluyó que la percepción del trabajador sobre la responsabilidad social tiene como principales indicadores el "Marketing responsable", "Valores y principios éticos" y la "Protección del medio ambiente". Sánchez (2010) refiere que el indicador "Economía" es el factor más destacado, seguido por la "Sociedad" y por último el "Medio Ambiente.

Castillo (2005, pág. 35) define responsabilidad social empresarial como "La obligación ética o moral, voluntariamente aceptada por la empresa como institución hacia la sociedad en conjunto, en reconocimiento y satisfacción de sus demandas o en reparación de los daños que puedan haberle sido causados a ésta en sus personas o en su patrimonio común por la actividad de la empresa". La Canadian Business for Social Responsability precisa que es: "El compromiso de una compañía de operar de manera económica y ambientalmente sostenible mientras reconoce los intereses los intereses de sus públicos de interés." En el Libro Verde de la Unión Europea (2001), se define como la "Integración voluntaria, por parte de las empresas, de las preocupaciones sociales y medioambientales en sus operaciones comerciales y sus relaciones con sus interlocutores".

Guedez (2006) precisa los elementos de la responsabilidad social empresarial, los cuales son compromiso de las empresas, decisión voluntaria, beneficios para la sociedad y públicos de interés, conducta ética, desempeño ambiental y adaptabilidad.

Para el presente trabajo de investigación se ha considerado las siguientes dimensiones para analizar la Responsabilidad Social Empresarial (Cajiga, 2013): balance social, balance ambiental, y balance económico, así como los servicios ofertados, localización, impresión general, personal y reputación.

\section{OBJETIVOS}

a) Conocer en qué medida el balance económico influye en la imagen institucional que perciben los clientes de las entidades financieras de Tacna, año 2017.

b) Identificar en qué medida el balance social influye en la imagen institucional que perciben los clientes de las entidades financieras de Tacna, año 2017.

c) Conocer en qué medida el balance ambiental influye en la imagen institucional que perciben los clientes de las entidades financieras de Tacna, año 2017. 


\section{METODOLOGÍA}

El tipo de investigación es básico (Hernández, Fernández y Baptista, 2014). El diseño es no experimental, de corte transversal, dado que la recolección de los datos se efectuará en un momento del tiempo. Tuvo como ámbito a los clientes de las principales entidades financieras que tienen agencias en la ciudad de Tacna durante el año 2017. Se consideró a 04 entidades financieras (bancos privados) que tienen mayor participación de mercado en la ciudad de Tacna, con un tamaño muestral de 381 clientes. La técnica utilizada fue la encuesta. Los instrumentos fueron validados a través del juicio de expertos. Se determinó la confiabilidad de los instrumentos utilizados mediante Alfa de Cronbach (0.892). Para el análisis general de las variables, se elaboró una escala de valoración en base a la Escala de Likert (entre 1 = muy en desacuerdo con lo afirmado en el ítem y $5=$ muy de acuerdo con lo afirmado en el ítem).

\section{RESULTADOS}

Respecto a "Responsabilidad social empresarial" la percepción de loa muestra encuestada fue:

Tabla 1

Dimensión "Balance económico"

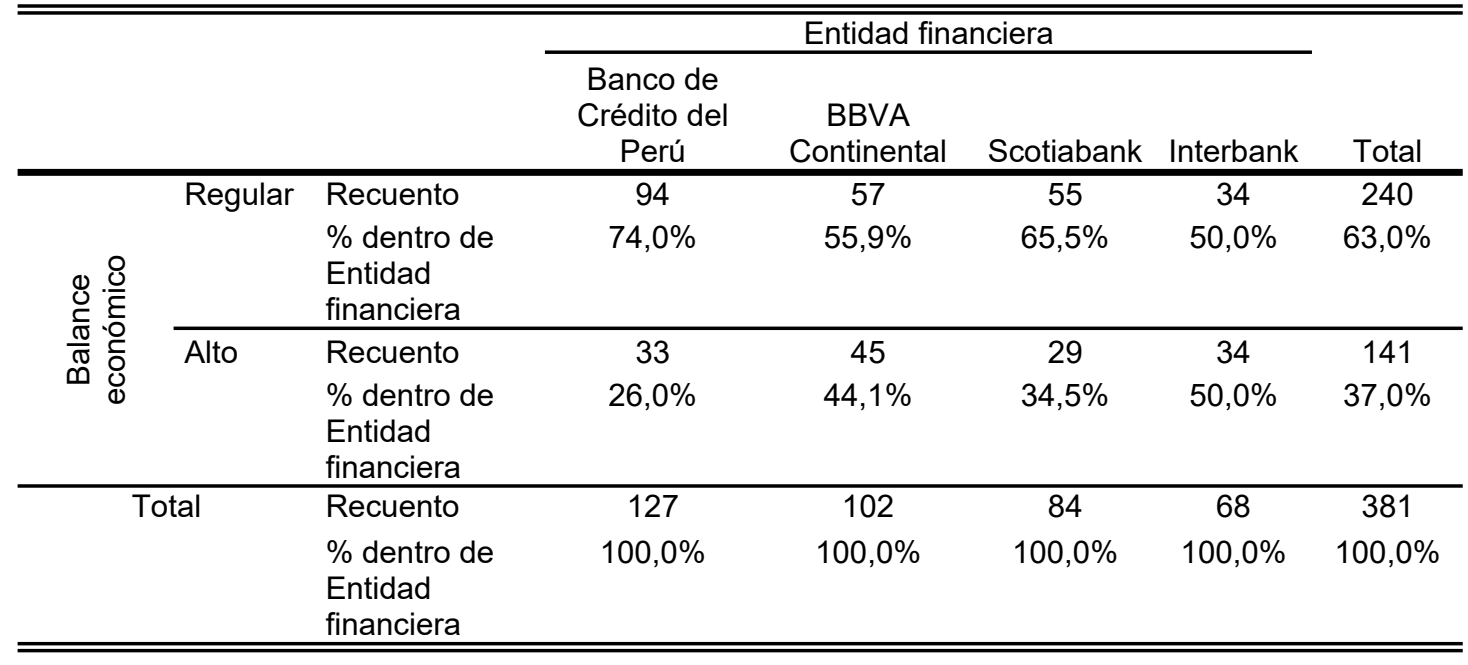

En la tabla 01, los resultados encontrados respecto a la dimensión "Balance económico", el $63,0 \%$ de los clientes considera que es de nivel regular el balance económico que perciben de las entidades financieras de Tacna, y el 37,0\% que es de nivel alto; siendo el Interbank la entidad cuyos clientes resaltan más dicha característica, seguido por el BBVA Continental, Scotiabank y finalmente el Banco de Crédito.

Respecto a la dimensión "Balance social" el 71,9\% de los clientes considera que es de nivel alto el balance social que perciben de las entidades financieras de Tacna, y el $28,1 \%$ que es de nivel regular; siendo el Scotiabank la entidad cuyos clientes resaltan más dicha característica, seguido por el BBVA Continental, Interbank y finalmente el Banco de Crédito del Perú. Respecto a la dimensión "Balance ambiental" el 83,2\% de los clientes considera que es de nivel regular el balance ambiental que perciben de las entidades financieras de Tacna, y el $16,8 \%$ 
que es de nivel bajo; siendo el Banco de Crédito del Perú la entidad cuyos clientes resaltan más dicha característica, seguido por el BBVA Continental, Interbank y finalmente el Scotiabank;

Después de efectuar el análisis de los resultados de las dimensiones, se analizó la variable "Responsabilidad social empresarial" de donde el 57,9\% de los clientes considera que es de nivel regular la responsabilidad social empresarial que perciben de las entidades financieras de Tacna, y el $42,1 \%$ que es de nivel alto; siendo el BBVA Continental la entidad cuyos clientes resaltan más dicha característica, seguido por el Interbank, Banco de Crédito del Perú y finalmente el Scotiabank; al analizar por dimensión, el aspecto más destacado por los clientes fue el "Balance social", siendo el aspecto a reforzar el "Balance ambiental" (Tabla 02).

Tabla 2

Variable "Responsabilidad social empresarial" (por dimensión)

\begin{tabular}{llcccc}
\hline \hline & & \multicolumn{4}{c}{ Entidad financiera } \\
\cline { 3 - 6 } & & $\begin{array}{c}\text { Banco de } \\
\text { Crédito del Perú }\end{array}$ & $\begin{array}{c}\text { BBVA } \\
\text { Continental }\end{array}$ & Scotiabank & Interbank \\
\cline { 2 - 6 } & Recuento & Recuento & Recuento & Recuento \\
\hline Balance económico & Bajo & 0 & 0 & 0 & 0 \\
& Regular & 94 & 56 & 55 & 34 \\
& Alto & 33 & 45 & 29 & 34 \\
Balance social & Bajo & 0 & 0 & 0 & 0 \\
& Regular & 40 & 31 & 15 & 21 \\
& Alto & 87 & 71 & 69 & 47 \\
& Bajo & 4 & 13 & 33 & 14 \\
& Regular & 123 & 89 & 51 & 54 \\
& Alto & 0 & 0 & 0 & 0 \\
\hline \hline
\end{tabular}

Se concluye con un nivel de confianza del $95 \%$, que la evidencia es significativa, de que, en los Bancos, existe una regular responsabilidad empresarial $(p<0.05)$

Respecto a las dimensiones de la variable "Imagen institucional":

Los resultados encontrados hacen referencia a la dimensión "Servicios ofertados" de donde el $85,0 \%$ de los clientes considera que es de nivel regular los servicios ofertados por las entidades financieras de Tacna, el 7,9\% que es de nivel alto, y el 7,1\% que es bajo; siendo el Scotiabank la entidad cuyos clientes resaltan más dicha característica,

Respecto a la dimensión "Localización", el 92,7\% de los clientes considera que es de nivel regular la localización de las entidades financieras de Tacna, y el 7,3\% que es adecuada; siendo el Banco de Crédito del Perú la entidad cuyos clientes resaltan más dicha característica, seguido por el BBVA Continental, Interbank y finalmente el Scotiabank;

Según la dimensión "Impresión general", el 80,3\% de los clientes considera que es de nivel elevado la impresión general que generan las entidades financieras de Tacna, y el 19,7\% que es regular; siendo el Banco de Crédito del Perú la entidad cuyos clientes resaltan más dicha característica, seguido por el BBVA Continental, Interbank y finalmente el Scotiabank.

Según la dimensión "Personal", el 86,9\% de los clientes considera que es de nivel regular las características del personal que labora en las entidades financieras de Tacna, y el 13,1\% que es 
adecuada; siendo el Scotiabank la entidad cuyos clientes resaltan más dicha característica, seguido por el Banco de Crédito del Perú, BBVA Continental y finalmente el Interbank.

Según la dimensión "Reputación" de donde el $88,7 \%$ de los clientes considera que es de nivel regular la reputación que caracteriza a las entidades financieras de Tacna, y el 11,3\% que es inadecuada; siendo el BBVA Continental la entidad cuyos clientes resaltan más dicha característica, seguido por el Interbank, Banco de Crédito del Perú, y finalmente el Scotiabank.

Después de efectuar el análisis de los resultados de las dimensiones, se analizó la variable "Imagen institucional" (Tabla 03), donde el 61,2\% de los clientes considera que es de nivel regular la imagen institucional que perciben de las entidades financieras de Tacna, y el $38,8 \%$ que es de nivel elevado; siendo el Interbank la entidad cuyos clientes resaltan más dicha característica, seguido por el BBVA Continental, Scotiabank y finalmente el Banco de Crédito del Perú

Tabla 3

Variable "Imagen institucional" (por dimensión)

\begin{tabular}{llcccc}
\hline \hline & & \multicolumn{4}{c}{ Entidad financiera } \\
\cline { 3 - 6 } & & $\begin{array}{c}\text { Banco de } \\
\text { Crédito del Perú }\end{array}$ & $\begin{array}{c}\text { BBVA } \\
\text { Continental }\end{array}$ & Scotiabank & Interbank \\
\cline { 3 - 6 } Servicios ofertados & Recuento & Recuento & Recuento & Recuento \\
\cline { 3 - 6 } & Regular & 17 & 5 & 0 & 5 \\
& Alto & 106 & 93 & 72 & 53 \\
Localización & 4 & 4 & 12 & 10 \\
& Bajo & 0 & 0 & 0 & 0 \\
Impresión general & Regular & 112 & 93 & 84 & 64 \\
& Alto & 15 & 9 & 0 & 4 \\
& Bajo & 0 & 0 & 0 & 0 \\
& Regular & 21 & 19 & 22 & 13 \\
& Alto & 106 & 83 & 62 & 55 \\
& Bajo & 0 & 0 & 0 & 0 \\
Reputación & Regular & 109 & 92 & 67 & 63 \\
& Alto & 18 & 10 & 17 & 5 \\
& Bajo & 13 & 8 & 14 & 8 \\
& Regular & 114 & 94 & 70 & 60 \\
& Alto & 0 & 0 & 0 & 0 \\
\hline \hline
\end{tabular}

Se concluye con un nivel de confianza del 95\%, que la evidencia es significativa, de que en los Bancos que forman parte del estudio, existe una regular "Imagen Institucional" $(p<0.05)$.

El balance económico no influye en la imagen institucional que perciben los clientes de las entidades financieras de Tacna (Tabla 04). El balance social si influye en la imagen institucional (Tabla 05) y el balance ambiental no influye en la imagen institucional ( $p: 0.749$ ) que perciben los clientes de las entidades financieras de Tacna, año 2017 a un nivel de 95\% de confianza en las 3 dimensiones.

Tabla 4 
Balance económico según Imagen institucional

\begin{tabular}{|c|c|c|c|c|}
\hline & & \multicolumn{2}{|c|}{ Imagen institucional } & \multirow[b]{2}{*}{ Total } \\
\hline & & Regular & Alto & \\
\hline \multirow[t]{2}{*}{ Balance económico } & Regular & 141 & 99 & 240 \\
\hline & Alto & 92 & 49 & 141 \\
\hline Total & & 233 & 148 & 381 \\
\hline
\end{tabular}

Tabla 05

Balance social según Imagen institucional

\begin{tabular}{clccc}
\hline \hline & & \multicolumn{2}{c}{ Imagen institucional } & \\
\cline { 3 - 4 } & & Regular & Alto & Total \\
\hline Balance social & Regular & 57 & 50 & 107 \\
& Alto & 176 & 98 & 274 \\
\multicolumn{2}{c}{ Total } & 233 & 148 & 381 \\
\hline \hline & & & & p: 0,048
\end{tabular}

Tabla 06

Balance ambiental según Imagen institucional

\begin{tabular}{|c|c|c|c|c|}
\hline & & \multicolumn{2}{|c|}{ Imagen institucional } & \multirow[b]{2}{*}{ Total } \\
\hline & & Regular & Alto & \\
\hline \multirow[t]{2}{*}{ Balance ambiental } & Bajo & 38 & 26 & 64 \\
\hline & Regular & 195 & 122 & 317 \\
\hline \multicolumn{2}{|l|}{ Total } & 233 & 148 & 381 \\
\hline
\end{tabular}

\section{DISCUSIÓN}

Después de analizar los resultados encontrados respecto al comportamiento de las variables investigadas desde la perspectiva del cliente del sistema financiero de Tacna, se tiene que existe influencia significativa de la responsabilidad social empresarial sobre la imagen institucional que perciben los clientes en el año 2017(p: 0,010); ello implica que las actividades que desarrollan las sucursales de los bancos en la región de Tacna que son afines a temas de responsabilidad social sí generan una buena aceptación en la población, y de forma especial en sus clientes, puesto que perciben que parte de las ganancias de la entidad, las devuelven a través de apoyos a gente con mayor necesidad o auspiciando eventos en fechas importantes. También se encontró que el $57,9 \%$ de los clientes considera que es de nivel regular la responsabilidad social empresarial que perciben de las entidades financieras de Tacna, siendo el aspecto más destacado por los clientes el "Balance social” y el aspecto a reforzar el "Balance ambiental"; y el $61,2 \%$ de los clientes considera que es de nivel regular la imagen institucional que perciben de las entidades financieras de Tacna, siendo el aspecto más destacado la "Impresión general" y el aspecto a reforzar la "Reputación". Dichos resultados concuerdan con los encontrados por Alvarado y Schlesinger (2008) en su trabajo la "Dimensionalidad de la Responsabilidad Social Empresarial Percibida y sus Efectos sobre la Imagen y Reputación: Una Aproximación desde el Modelo de Carroll", que concluye que las acciones y programas de 
responsabilidad social empresarial podrían mejorar la imagen y la reputación de las empresas ante los ojos de sus consumidores y ser una importante fuente de ventaja competitiva para las mismas; y con los resultados de Sánchez (2010) en su trabajo "La responsabilidad social empresarial y su relación con el servicio percibido por los clientes de la institución financiera Interbank de Tacna, en el 2010", quien concluye que la percepción del cliente sobre la responsabilidad social empresarial que caracteriza a la entidad financiera fue regular, el "Servicio al cliente" es de un servicio regular, y que existe relación directa y significativa entre la responsabilidad social empresarial y el servicio percibido por los clientes.

Es recomendable que los directivos de las agencias estudiadas fortalezcan su estrategia de responsabilidad social empresarial, centren principalmente su atención en las actividades afines a temas sociales, como el apoyo a las poblaciones vulnerables de la región. Asimismo, considerar el ofrecer al cliente condiciones más flexibles para que puedan cumplir con sus obligaciones de préstamos y de morosidad, a través de incrementar el número de campañas financieras en el año e incrementar los auspicios en las actividades educativas, de salud, culturales y apoyando de forma más directa las campañas de reducción de la contaminación o de reciclaje que las entidades públicas impulsan.

\section{CONCLUSIONES}

La responsabilidad social empresarial influye en la imagen institucional que perciben los clientes de las entidades financieras de Tacna. El balance económico no influye en la imagen institucional pero sí el balance social. El balance ambiental no influye en la imagen institucional percibida por los usuarios de las instituciones trabajadas.

\section{BIBLIOGRAFÍA}

Cajiga, J. (2013). Cemefi. Centro Mexicano para la Filantropía. El Concepto De Responsabilidad Social Empresarial: Disponible en. <http://www.cemefi.org/esr/images/stories/pdf/esr/concepto_esr.pdf>

Carroll, A.y Shabana, K. (2010). The business case for corporate social responsibility: a review of concepts, research and practice. International Journal of Management Reviews, Vol. 12, No. 1 , pp. 85-105.

Castillo, C. (2005). La responsabilidad social de la empresa y los consumidores. Presentado en el Encuentro Nacional de Organizaciones de Consumidores. Buenos Aires (Argentina).

Cea, R. (2010). La responsabilidad social corporativa en las entidades bancarias de la Unión Europea: Análisis empírico y propuesta de modelo normalizado; Universidad Autónoma de Madrid. Disponible en: https://repositorio.uam.es/xmlui/bitstream/handle/10486/4183/28409_cea_moure_r amiro.pdf? sequence $=1 \&$ isAllowed $=y$

Guedez, V. (2006). Ética y práctica de la responsabilidad social empresarial. Venezuela: Editorial Planeta.

Harjoto, M., Laksmana, I., y Lee, R. (2015). Board diversity and corporate social responsibility. Journal of Business Ethics, 132(4), 641-660.

Hernández, R.; Fernández, C. y Baptista, M. (2010). Metodología de la investigación. México: McGraw-Hill Educación. 5a Edición. 
Lizarzaburu, E. y Del Brío, J. (2016). Responsabilidad Social Corporativa y Reputación Corporativa en el sector financiero de países en desarrollo. Disponible mes: <https://gcg.universia.net/article/viewFile/1825/1839>

Marquina, P. (2015). Impacto de la responsabilidad social empresarial en el comportamiento de compra y disposición a pagar de consumidores bogotanos. Artículo de Sciencie Direc. Disponibler

en: <http://www.sciencedirect.com/science/article/pii/S0123592315000558>

Marquina, P. (2012). La influencia de la responsabilidad social empresarial en el comportamiento de compra de los consumidores peruanos. Tesis para optar al grado de Doctor en Administración Estratégica de Empresas de la PUCP. http://tesis.pucp.edu.pe/repositorio/handle/123456789/1606

Mercado, P. y García, P. (2007). La responsabilidad social en empresas del Valle de Toluca (México). Un estudio exploratorio. Artículo de Scielo. Disponible en: $<$ http://www.scielo.org.co/scielo.php?pid=S0123$59232007000100005 \&$ script=sci_arttext\&tIng=en>

Pérez - Aranda, J. (2016). Valoración de la responsabilidad social empresarial (RSE) por la demanda hotelera; de la Universidad Jaume I (España). Disponible en: https://www.tesisenred.net/bitstream/handle/10803/395950/2016_Tesis_PerezAranda_Antonio.pdf?sequence $=1$ \&isAllowed $=y$

Quihue, G. (2011). La responsabilidad social que caracteriza a la Caja Municipal de Ahorro y Crédito S.A. de Tacna, desde la perspectiva de sus clientes internos y externos. Tesis para optar al grado académico de Magíster en Administración y Dirección de Empresas de la Universidad Privada de Tacna.

Sánchez, J. (2010). La responsabilidad social empresarial y su relación con el servicio percibido por los clientes de la institución financiera Interbank de Tacna, en el 2010. Tesis para optar al título profesional de Ingeniero Comercial de la Universidad Privada de Tacna. 